\title{
Description of the unknown males of three Chinese Meligethes (Coleoptera: Nitidulidae, Meligethinae)
}

\author{
Simone SABATELLI ${ }^{1}$, Paolo AUDISIO ${ }^{1}$, Josef JELÍNEK ${ }^{2}$, Meike LIU ${ }^{3,4}$, Min HUANG ${ }^{3, *}$ \\ ${ }^{1}$ Department of Biology and Biotechnologies “C. Darwin”, Sapienza Università degli Studi di Roma - Viale dell'Università 32, Rome \\ I-00185, Italy - simone.sabatelli@uniroma1.it; paolo.audisio@uniroma1.it \\ ${ }^{2}$ Department of Entomology, National Museum - Circusová 1740, CZ - 19300 Praha 9, Horní Počernice, Czech Republic \\ jj.nitidula@seznam.cz \\ ${ }^{3}$ Key Laboratory of Plant Protection Resources and Pest Management of Ministry of Education, Entomological Museum, Northwest \\ A\&F University - Yangling, Shaanxi 712100, China - huangmin@nwsuaf.edu.cn \\ ${ }^{4}$ College of Agriculture, Yangtze University - Jingzhou, Hubei 434025, China - liumk2009@126.com \\ * Corresponding author
}

\begin{abstract}
In this paper we present the description of male genitalia and additional information on taxonomy of three recently described and poorly known Chinese endemic members of the genus Meligethes Stephens, 1830, Meligethes (Odonthogethes) brassicogethoides Audisio, Sabatelli \& Jelínek, 2015, M. (O.) inexpectatus Liu, Huang, Cline, Sabatelli \& Audisio, 2017, and M. (O.) tricuspidatus Liu, Huang, Cline \& Audisio, 2018.
\end{abstract}

Key words: pollen beetles, male genitalia, China.

\section{Introduction}

The pollen beetle genus Meligethes (Nitidulidae, Meligethinae), established by Stephens in 1830, as re-defined and delimited by Audisio et al. (2009) and Trizzino et al. (2009), was recently revised by Audisio et al. (2015). Subsequently, nine additional new species from China were described by Chen et al. (2015) and by Liu et al. $(2016,2017,2018)$, and a few others are still under scrutiny, or under description elsewhere. This genus now officially includes 62 species, mostly distributed in the Eastern portion of the Palaearctic Region. Larvae of all members of the genus are associated with flowers of Rosaceae. Recent molecular analyses (Trizzino et al. 2009; Liu et al. 2020) fully confirmed an almost certainly monophyletic lineage including the taxa Meligethes Stephens, 1830 (s. str.), Odonthogethes Reitter, 1871 (a subgenus of the former), Brassicogethes Audisio \& Cline, 2009, and Meligethinus Grouvelle, 1909. This article includes the descriptions of the previously unknown males of Meligethes (Odonthogethes) brassicogethoides Audisio, Sabatelli \& Jelínek, 2015 (known thus far from Yunnan), M. (O.) inexpectatus Liu, Huang, Cline, Sabatelli \& Audisio, 2017 (known thus far from Sichuan), and $M$. (O.) tricuspidatus Liu, Huang, Cline \& Audisio, 2018 (known thus far from Hubei), based on recently collected material from Central and S China.

\begin{abstract}
Abbreviations
Acronyms of museum institutions:

CAR-MZUR P. Audisio's collection, currently housed in the Zoological Museum, Sapienza Rome University, Rome, Italy

NMPC National Museum, Prague, Czech Republic NWAU Entomological Museum of the Northwest A\&F University, Yangling, Shaanxi, China
\end{abstract}

\section{Meligethes (Odonthogethes) brassicogethoides}

Audisio, Sabatelli \& Jelínek, 2015 (Figs 1, 4-5)

This species was described only on the base of two female specimens collected in SW China, Yunnan province (Audisio et al. 2015). A recent collection from SW China, Guizhou province, allowed us to compare a short series of Odonthogethes specimens of both sexes, the females exhibiting a rather peculiar ovipositor, identical to that known in $M$. (O.) brassicogethoides from the neighbor Yunnan, despite their body shape (Fig. 1) was distinctly less elongate than in the type material.

The unknown male genitalia of this species as in Figs 4-5. They are characterized by a standard wide and short tegmen, with widely U-shaped medial excision and rather narrow and pointed paramera, associated with middlesized aedeagus, obtusely and uniformly triangularly pointed distad. 

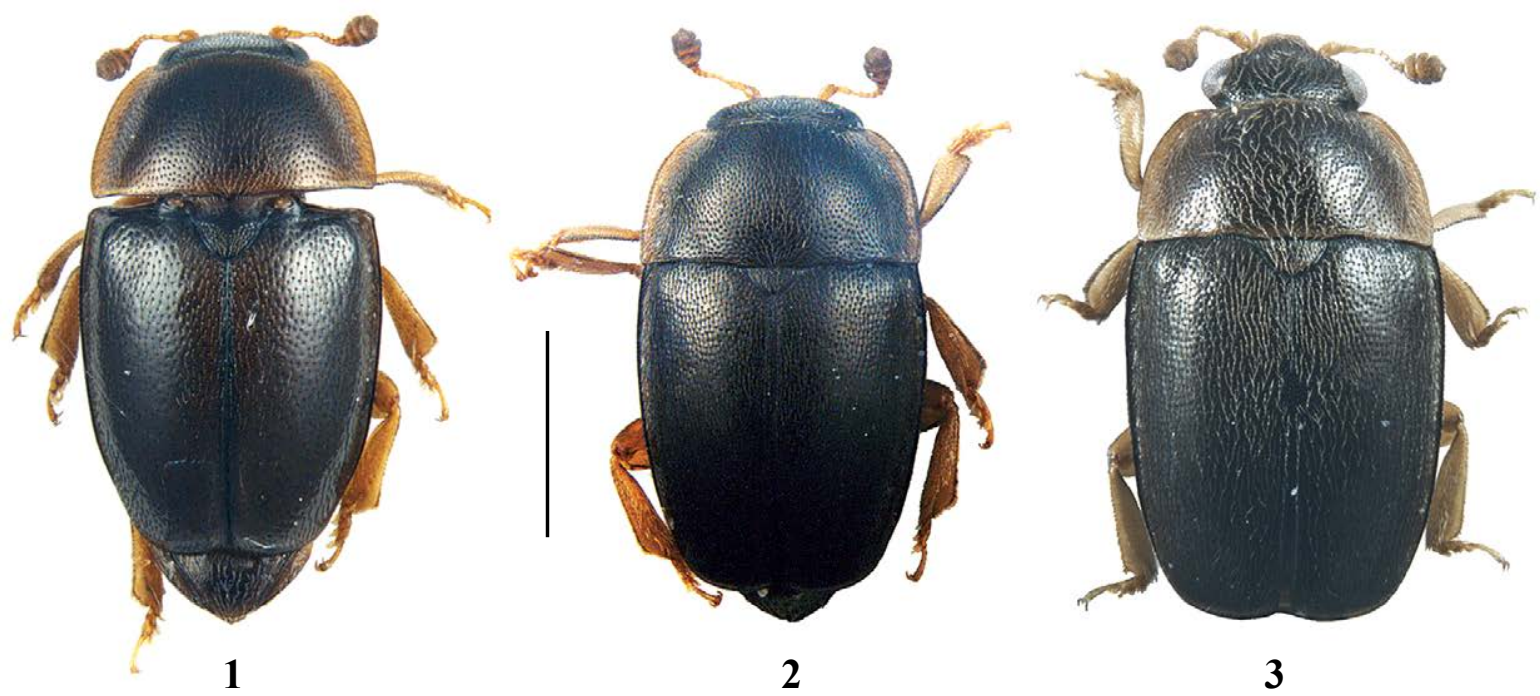

Figs 1-3 - Dorsal habitus of males of Meligethes (Odonthogethes) spp. 1, M. brassicogethoides from Guizhou; 2, M. inexpectatus from Hubei; 3, M. tricuspidatus from Shaanxi. Scale bar: $1 \mathrm{~mm}$.

Taxonomic remarks. The shape of the male genitalia in this species is unusual within members of the $M$. (O.) chinensis Kirejtshuk species-group (Audisio et al. 2015), most included taxa of this lineage exhibiting, in fact, an aedeagus chisel-shaped distad.

Examined Material. China: Guizhou, Qiandongnan City, Leigong Mts, 18.VII.2018, Chen lgt., on flowering shrubs of Artemisia lactiflora Wall. ex DC. (Asteraceae), 2 え ${ }^{\lambda}$, 2 우 (NWAU, CAR-MZUR); Shaanxi, Haoping Temple, 30.IV.2017, Cao lgt, 2 우우 (NWAU, CAR-MZUR).

\section{Meligethes (Odonthogethes) inexpectatus}

Liu, Huang, Cline, Sabatelli \& Audisio, 2017

(Figs 2, 6-7)

This species was described only on the base of a female specimen collected in SW China, Sichuan province (Liu et al. 2017). This isolated female was previously erroneously believed as representing the unknown female of another member of the $O$. chinensis species-group, $O$. scrobescens Chen, Lin, Huang \& Yang, 2015 from Sichuan (Liu et al. 2016). A recent collection from SW China, Hubei province (see below), allowed us to compare a short series of specimens of both sexes, the females exhibiting an ovipositor identical to that known in $O$. inexpectatus from Sichuan.

The unknown male genitalia as in Figs 6-7. They are characterized by a standard tegmen, with widely $\mathrm{V}$-shaped medial excision, and paramera obtusely pointed distad, associated with peculiarly long and large aedeagus, the latter widely chisel-shaped distad.

Taxonomic remarks. The shape of the male genitalia seems to confirm the placement of this species well within the $M$. (O.) chinensis species-group (Audisio et al. 2015), where it appears to be characterized by an aedeagus peculiarly large, long, and widely chisel-shaped distad (Fig. 7).

Examined Material. China: Sichuan, E of Luding, Erlang Mts (Erlangshan), 2600-2700 m a.s.1., 14/15.VI.2003, S. Murzin lgt., $2 \widehat{\partial}$ (NMPC, CAR-MZUR); Shaanxi, Ankang, Langao, NanGong Mts, 26.VI.2018, Yang lgt, 1 $\widehat{o}$ (NWAU); Hubei, Xiangyang, Baokang, Houping Town, Hengchong Scenic Spot, 06.VII.2018, Yang lgt, on flowering shrubs of Astilbe chinensis (Maxim.) Franch. et Savat. (Saxifragaceae), 1 o, 1 \& (NWAU).

\section{Meligethes (Odonthogethes) tricuspidatus}

Liu, Huang, Cline \& Audisio, 2018 (Figs 3, 8-9)

This species was described only on the base of three female specimens collected in Central China, Hubei province (Liu et al. 2018). A recent collection from SW China, N Sichuan province (see below), allowed us to compare a couple of specimens of both sexes, the female exhibiting the same, very peculiarly shaped ovipositor known in $O$. tricuspidatus from Hubei.

The unknown male genitalia of this species as in Figs $8-9$. They are characterized by a standard short and wide tegmen, with paramera obtuse distad, associated with a middle-sized aedeagus, narrowly chisel-shaped distad.

Taxonomic remarks. The shape of the male genitalia seems to confirm the placement of this species well within the $M$. (O.) chinensis species-group (Audisio et al. 2015).

Examined Material. China: N Sichuan, XiaoZhaizi National Nature Reserve, $4 \mathrm{Km}$ NE of Qingpianxiang, Zheng-

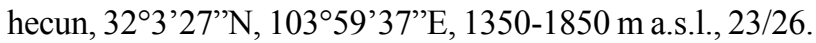




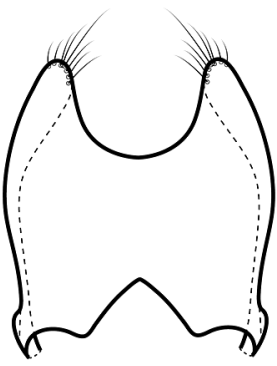

4
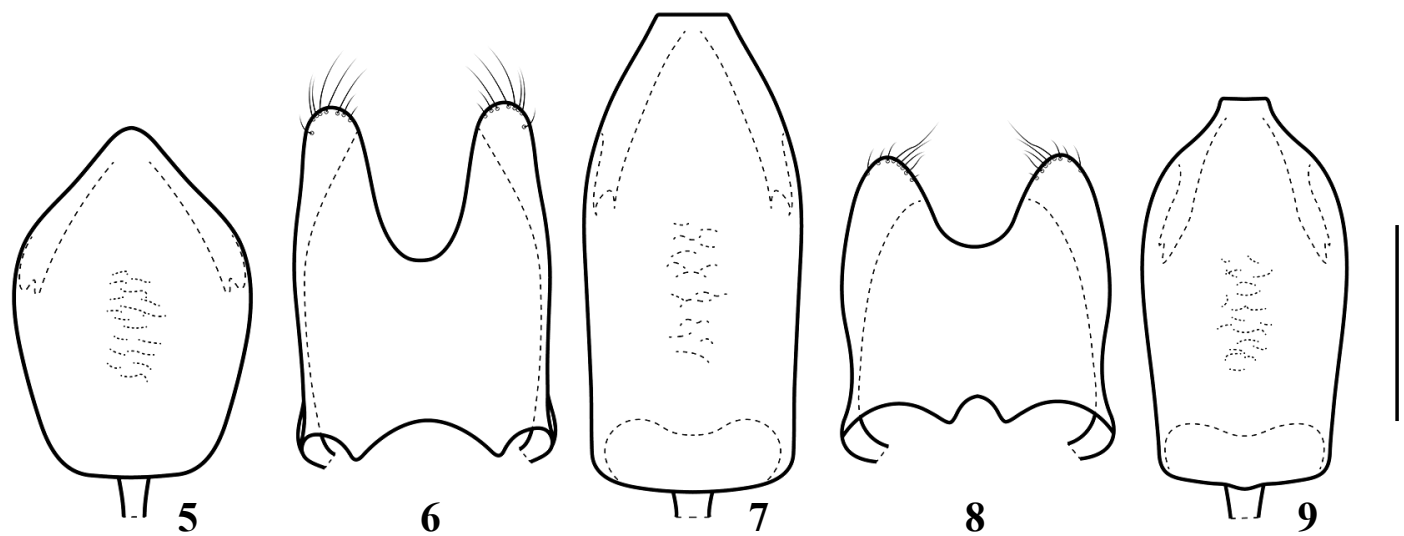

Figs 4-9 - Male genitalia (tegmen and aedeagus, dorsal view) of Meligethes (Odonthogethes) spp. 4-5, M. brassicogethoides from Guizhou; 6-7, M. inexpectatus from Hubei; 8-9, M. tricuspidatus from Shaanxi. Scale bar $=0.2 \mathrm{~mm}$.

VI.2017, P. Kabátek lgt, 1 ð, 1 \& (NMPC, CAR-MZUR); Hubei, Shennongjia Scenic Area, ca. 2500 m, 16.VI.2017, P. Audisio lgt, on flowering shrubs of Rubus rosaefolius Smith (Rosaceae), 1 \& (NWAU); Shaanxi, Ankang, Langao, NanGong Mts, 26.VI.2018, Yang lgt, on flowering shurbs of Spiraea salicifolia L. (Rosaceae), 1 o, 1 q (NWAU).

Bionomical notes. No sure larval-host plant relationships are known for this species, although the above cited $R u$ bus rosaefolius Smith (Rosaceae) and Spiraea salicifolia L. (Rosaceae) could both represent possible alternative hosts.

Acknowledgements - This research was supported by funds from the State Administration of Foreign Experts Affair for Recruitment Program of High-end Foreign Experts (Popular Republic of China). The authors greatly appreciate the valuable comments on an earlier version of this manuscript by an anonymous reviewer. The author M.L. thanks the China Scholarship Council for financial support for her stay in Rome (November, 2016-December, 2017), in collaboration with coauthor P.A.

\section{References}

Audisio P., Cline A.R., De Biase A., Antonini G., Mancini E., Trizzino M., Costantini L., Strika S., Lamanna F., Cerretti P. 2009. Preliminary re-examination of genus-level taxonomy of the pollen beetle subfamily Meligethinae (Coleoptera: Nitidulidae). Acta Entomologica Musei Nationalis Pragae, 49 (2): 341-504.

Audisio P., Sabatelli S., Jelínek J. 2015. Revision of the pollen beetle genus Meligethes Stephens, 1830 (Coleoptera: Nitid- ulidae). Fragmenta entomologica, 46 (2014): 19-112. Doi: http://dx.doi.org/10.4081/fe.2014.71

Chen Y., Lin X.L., Huang M., Yang X.K. 2015. A new species of Lamiogethes and a new species of Meligethes from China (Coleoptera: Nitidulidae: Meligethinae). Zootaxa, 3999 (3): 413-420. Doi: http://dx.doi.org/10.11646/zootaxa.3999.3.6

Liu M., Huang M., Cline A.R., Audisio P. 2018. New and poorly known Meligethes Stephens from China, with bionomical data on some species (Coleoptera: Nitidulidae: Meligethinae). Zootaxa, 4392: 546-566. Doi: https://doi.org/10.11646/ zootaxa.4392.3.6

Liu M., Huang M., Cline A.R., Mancini E., Scaramuzzi A., Paradisi S., Audisio P., Badano D., Sabatelli S. 2020. Rosaceae, Brassicaceae and Pollen Beetles: exploring relationships and evolution in an anthophilous beetle lineage (Nitidulidae, Meligethes-complex of genera) using an integrative approach. Frontiers in Zoology, In Review, Doi: 10.21203/ rs.3.rs-37852/

Liu M.K., Huang M., Cline A.R., Sabatelli S., Audisio P. 2017. A new species of Meligethes Stephens from China and additional data on members of the $M$. chinensis species-complex (Coleoptera: Nitidulidae, Meligethinae). Fragmenta entomologica, 49 (1): 79-84. Doi: http://dx.doi.org/10.4081/ fe. 2017.234

Liu M.K., Yang X.K., Huang M., Jelínek J., Audisio P. 2016. Four new species of Meligethes from China and additional data on other species of the genus (Coleoptera: Nitidulidae: Meligethinae). Zootaxa, 4121 (2): 101-116. Doi: http:// dx.doi.org/10.11646/zootaxa.4121.2.1

Trizzino M., Audisio P., Antonini G., De Biase A., Mancini E. 2009. Comparative analysis of sequences and secondary structures of the rRNA internal transcribed spacer 2 (ITS2) in pollen-beetles of the subfamily Meligethinae (Coleoptera, Nitidulidae): potential use of slippage-derived sequences in molecular systematics. Molecular Phylogenetics and Evolution, 51 (2): 215-226. Doi: http://dx.doi.org/10.1016/j. ympev.2008.11.004 